\title{
APPROXIMATION PROPERTIES FOR DUAL SPACES
}

\author{
VEGARD LIMA
}

\begin{abstract}
We prove that a Banach space $X$ has the metric approximation property if and only if $\mathscr{F}(Y, X)$ is an ideal in $\mathscr{L}\left(Y, X^{* *}\right)$ for all Banach spaces $Y$. Furthermore, $X^{*}$ has the metric approximation property if and only if for all Banach spaces $Y$ and all Hahn-Banach extension operators $\phi$ : $X^{*} \rightarrow X^{* * *}$ there exists a Hahn-Banach extension operator $\Phi: \mathscr{F}(Y, X)^{*} \rightarrow \mathscr{L}\left(Y, X^{* *}\right)^{*}$ such that $\Phi\left(x^{*} \otimes y^{* *}\right)=\left(\phi x^{*}\right) \otimes y^{* *}$ for all $x^{*} \in X^{*}$ and all $y^{* *} \in Y^{* *}$. We also prove that $X^{*}$ has the approximation property if and only if for all Banach spaces $Y$ and all HahnBanach extension operators $\phi: X^{*} \rightarrow X^{* * *}$ there exists a Hahn-Banach extension operator $\Phi: \mathscr{F}(Y, X)^{*} \rightarrow \mathscr{W}\left(Y, X^{* *}\right)^{*}$ such that $\Phi\left(x^{*} \otimes y^{* *}\right)=\left(\phi x^{*}\right) \otimes y^{* *}$ for all $x^{*} \in X^{*}$ and all $y^{* *} \in Y^{* *}$, which in turn is equivalent to $\mathscr{F}(Y, \hat{X})$ being an ideal in $\mathscr{W}\left(Y, \hat{X}^{* *}\right)$ for all Banach spaces $Y$ and all equivalent renormings $\hat{X}$ of $X$.
\end{abstract}

\section{Introduction}

In [2] Godefroy, Kalton, and Saphar introduced and studied the notion of an ideal. Recall that if $X$ is a closed subspace of a Banach space $Y$, then $X$ is an ideal in $Y$ if the annihilator of $X$ in $Y^{*}, X^{\perp}$, is the kernel of a norm one projection on $Y^{*}$. If $X$ is a subspace of a normed space $Y$, then we say that $X$ is an ideal in $Y$ if the norm closure of $X, \bar{X}$, is an ideal in $\bar{Y}$.

A linear operator $\phi: X^{*} \rightarrow Y^{*}$ is called a Hahn-Banach extension operator if $\left(\phi x^{*}\right)(x)=x^{*}(x)$ and $\left\|\phi x^{*}\right\|=\left\|x^{*}\right\|$ for all $x \in X$ and $x^{*} \in X^{*}$. The set of norm-preserving extensions of $x^{*} \in X^{*}$ is denoted $\mathrm{HB}\left(x^{*}\right)$, and we denote the set of all Hahn-Banach extension operators $\phi: X^{*} \rightarrow Y^{*}$ by $\mathrm{B}(X, Y)$. It is easy to see that $X$ is an ideal in $Y$ if and only if $\mathrm{B}(X, Y) \neq \emptyset$. Indeed, if $P$ is a norm-one projection on $Y^{*}$ such that $X^{\perp}=\operatorname{ker} P$, and if $i: X \rightarrow Y$ is the identity map, then $P=\phi \circ i^{*}$ for some $\phi \in \mathrm{BB}(X, Y)$.

In [9] (cf. Theorem 5.2) Lima, Nygaard, and Oja obtained the following characterization of the metric approximation property for dual spaces.

Theorem. Let $X$ be a Banach space. The following statements are equivalent.

(a) $X^{*}$ has the metric approximation property. 
(b) For all Banach spaces $Y$, there exists a $\Phi \in H(\mathscr{F}(X, Y), \mathscr{L}(X, Y))$ such that

$$
\Phi\left(y^{*} \otimes x^{* *}\right)(T)=\left(y^{*} \otimes x^{* *}\right)(T)
$$

for all $x^{* *} \in X^{* *}, y^{*} \in Y^{*}$, and $T \in \mathscr{L}(X, Y)$.

(c) There exists a $\Phi \in H\left(\mathscr{F}(X, X), \operatorname{span}\left(\mathscr{F}(X, X),\left\{I_{X}\right\}\right)\right)$ such that

$$
\Phi\left(x^{*} \otimes x^{* *}\right)(T)=\left(x^{*} \otimes x^{* *}\right)(T)
$$

for all $x^{*} \in X^{*}, x^{* *} \in X^{* *}$, and $T \in \operatorname{span}\left(\mathscr{F}(X, X),\left\{I_{X}\right\}\right)$.

In [14] Lima and Lima proved that a Banach space $X$ has the metric approximation property if and only if $\mathscr{F}(Y, X)$ is an ideal in $\mathscr{L}(Y, X)$ for all Banach spaces $Y$. In this paper we shall look for conditions on $X$ that ensure that $\mathscr{F}(Y, X)$ is an ideal in $\mathscr{L}\left(Y, X^{* *}\right)$, resp. $\mathscr{W}\left(Y, X^{* *}\right)$, for all Banach spaces $Y$. In particular, we will be interested in associated Hahn-Banach extension operators with "nice" properties as in the theorem above.

This paper consists of 4 sections. In Section 2 we study the metric approximation property for a Banach space $X$ and its relation to ideals of operators. More specifically, we are interested in the space $\mathscr{L}\left(Y, X^{* *}\right)$ and Hahn-Banach extension operators from $\mathscr{F}(Y, X)^{*}$ to $\mathscr{L}\left(Y, X^{* *}\right)^{*}$ for Banach spaces $Y$. We show that the metric approximation property for $X$ is equivalent to the existence of a $\phi \in \mathrm{B}\left(X, X^{* *}\right)$ and a $\Phi \in \mathrm{B}\left(\mathscr{F}(Y, X), \mathscr{L}\left(Y, X^{* *}\right)\right)$ such that $\Phi\left(x^{*} \otimes y\right)(T)=\left(\phi x^{*} \otimes y\right)(T)$ for all $x^{*} \in X^{*}, y \in Y$, and $T \in \mathscr{L}\left(Y, X^{* *}\right)$ (see Theorem 2.7).

In Section 3 we prove, in Theorem 3.10, that $X^{*}$ has the metric approximation property if and only if for all $\phi \in \mathrm{B}\left(X, X^{* *}\right)$ and all Banach spaces $Y$ there exists a $\Phi \in \mathrm{B}\left(\mathscr{F}(Y, X), \mathscr{L}\left(Y, X^{* *}\right)\right)$ such that

$$
\Phi\left(x^{*} \otimes y^{* *}\right)(T)=\left(\phi x^{*} \otimes y^{* *}\right)(T)
$$

for all $x^{*} \in X^{*}, y^{* *} \in Y^{* *}$, and all $T \in \mathscr{L}\left(Y, X^{* *}\right)$. If we replace $\Phi \in$ $\mathrm{B}\left(\mathscr{F}(Y, X), \mathscr{L}\left(Y, X^{* *}\right)\right)$ above with $\Phi \in \mathrm{B}\left(\mathscr{F}(Y, X), \mathscr{W}\left(Y, X^{* *}\right)\right)$, then we get a property which is equivalent to the approximation property for $X^{*}$ (see Theorem 3.7).

In Section 4 we show why there is no mention of the approximation property in Section 2. We show that a result similar to Lemma 2.1 does not exist for the approximation property.

Let us fix some notation. We let $\mathscr{F}(Y, X), \mathscr{K}(Y, X), \mathscr{W}(Y, X)$ and $\mathscr{L}(Y, X)$ denote the Banach space of all finite rank, compact, weakly compact and bounded operators, respectively, whenever $X$ and $Y$ are Banach spaces. $I_{X}$ denotes the identity operator on $X$. If $X$ is a Banach space then $B_{X}$ is the closed unit ball of $X$ and $S_{X}$ is the unit sphere of $X$. For a set $Z \subset X$, its 
norm closure is denoted by $\bar{Z}$, its linear span by $\operatorname{span} Z$, and its convex hull by conv $Z$.

We consider only Banach spaces over the real field $R$.

\section{Metric approximation property and ideals of operators}

In [6, Lemma 1] Johnson proved that $\mathscr{F}(Y, X)$ is an ideal in $\mathscr{L}(Y, X)$ for all Banach spaces $Y$ whenever $X$ has the metric approximation property. In [14] it was proved that these two conditions are in fact equivalent.

Our first result is a slight improvement of the metric version of Johnson's result.

Lemma 2.1. Let $X$ be a Banach space. If $X$ has the metric approximation property [resp. metric compact approximation property], then $\mathscr{F}(Y, X)$ [resp. $\mathscr{K}(Y, X)]$ is an ideal in $\mathscr{L}\left(Y, X^{* *}\right)$ for every Banach space $Y$.

Proof. We only show the implication for the metric approximation property, the case with compact operators is similar.

Assume $X$ has the metric approximation property. Let $\left(T_{\alpha}\right) \subseteq \mathscr{F}(X, X)$ be a net such that $\sup _{\alpha}\left\|T_{\alpha}\right\| \leq 1$ and $T_{\alpha} \rightarrow I_{X}$ uniformly on compact subsets of $X$.

Let $Y$ be a Banach space. Define $U_{\alpha}: \mathscr{L}\left(Y, X^{* *}\right) \rightarrow \mathscr{F}(Y, X)$ by

$$
U_{\alpha}(T)=T_{\alpha}^{* *} \circ T .
$$

We have $\left\|U_{\alpha}\right\| \leq\left\|T_{\alpha}\right\| \leq 1$.

Let $G \subseteq \mathscr{L}\left(Y, X^{* *}\right)$ be finite dimensional, and let $F=G \cap \mathscr{F}(Y, X)$. Let $\varepsilon>0$, and let $\left\{T_{i}\right\}_{i=1}^{n}$ be an $\varepsilon / 3$-net for $B_{F}$. Letting

$$
K=\bigcup_{i=1}^{n} \overline{T_{i}\left(B_{Y}\right)},
$$

we see that $K$ is a compact subset of $X$. Choose $\alpha_{0}$ such that $\sup _{x \in K} \| x-$ $T_{\alpha_{0}} x \|<\varepsilon / 3$. Then $\left\|T_{i}-U_{\alpha_{0}}\left(T_{i}\right)\right\|<\varepsilon / 3$ for $i=1, \ldots, n$, and thus $\| T-$ $U_{\alpha_{0}}(T) \|<\varepsilon$ for all $T \in B_{F}$. By the local formulation of ideals (see [1], or f.ex. [7] or [8]) this implies that $\mathscr{F}(Y, X)$ is an ideal in $\mathscr{L}\left(Y, X^{* *}\right)$.

Remark 2.2. Every Banach space $X$ is an ideal in its bidual $X^{* *}$, and the natural embedding $i: X^{*} \rightarrow X^{* * *}$ is a Hahn-Banach extension operator, $i \in \mathrm{B}\left(X, X^{* *}\right) . U_{\alpha}$ in the proof above may be written as $U_{\alpha}(T)=T_{\alpha}^{* *} \circ i^{*} \circ T$, and it is clear that we may replace $X^{* *}$ in the lemma above with any Banach space containing $X$ as an ideal.

Next we will extend some results from [14] in order to prove Theorem 2.7, which says that a Banach space $X$ has the metric approximation property if 
and only if there exists a $\phi \in \mathrm{B}\left(X, X^{* *}\right)$ such that, for every Banach space $Y$, there is a Hahn-Banach extension operator $\Phi \in \mathrm{B}\left(\mathscr{F}(Y, X), \mathscr{L}\left(Y, X^{* *}\right)\right)$ satisfying (\#) for all $x^{*} \in X^{*}, y \in Y$, and $T \in \mathscr{L}\left(Y, X^{* *}\right)$. We will prove the next results for closed operator ideals $\mathscr{A}$ and $\mathscr{B}$, in the sense of Pietsch [17]. Recall that, in particular, this means that $\mathscr{F}(Y, X) \subseteq \mathscr{A}(Y, X)$ and $\mathscr{A}(Y, X)$ is a closed subspace of $\mathscr{L}(Y, X)$.

Lemma 2.3. Let $X$ and $Y$ be Banach spaces, and let $Z$ be a subspace of $Y$ such that the norm of $Y$ is locally uniformly rotund at every point of $Z$. Let $\mathscr{A}$ and $\mathscr{B}$ be closed operator ideals satisfying $\mathscr{A} \subseteq \mathscr{B}$. If $\Phi \in$ $H\left(\mathscr{A}(Y, X), \mathscr{B}\left(Y, X^{* *}\right)\right)$, then there is a $\phi \in H\left(X, X^{* *}\right)$ such that

$$
\Phi\left(x^{*} \otimes z\right)(T)=\left(\phi x^{*} \otimes z\right)(T)
$$

for all $x^{*} \in X^{*}, z \in Z$, and $T \in \mathscr{B}\left(Y, X^{* *}\right)$.

Proof. Using the local uniform rotundity modulus of $Y$ (see e.g. [16, p. 460]) it is easy to show that every $z \in S_{Z}$ is is a weak*-denting point in $B_{Y^{* *}}$. Thus we may use Lemma 3.1 in [11], and get that for all $x^{*} \in X^{*}$ and $z \in Z$

$$
\mathrm{H}\left(x^{*} \otimes z\right)=\mathrm{B}\left(x^{*}\right) \otimes\{z\} .
$$

Given $z \in Z$ we choose a $z^{*} \in Y^{*}$ such that $z^{*}(z)=1$. Using (1) we may define a Hahn-Banach extension operator $\phi_{z} \in \mathrm{B}\left(X, X^{* *}\right)$ by letting $\left(\phi_{z} x^{*}\right)\left(x^{* *}\right)=$ $\Phi\left(x^{*} \otimes z\right)\left(z^{*} \otimes x^{* *}\right)$ for all $x^{* *} \in X^{* *}$. Thus for all $z \in Z$ there is a $\phi_{z} \in$ $\mathrm{B}\left(X, X^{* *}\right)$ such that

$$
\Phi\left(x^{*} \otimes z\right)(T)=\left(\phi_{z} x^{*} \otimes z\right)(T)
$$

for all $x^{*} \in X^{*}$ and $T \in \mathscr{B}\left(Y, X^{* *}\right)$.

Following [13], given $\Phi \in \mathrm{B}\left(\mathscr{A}(Y, X), \mathscr{B}\left(Y, X^{* *}\right)\right)$ and $\phi \in \mathrm{B}\left(X, X^{* *}\right)$, we will write

$$
Y_{\Phi \phi}=\left\{y \in Y: \Phi\left(x^{*} \otimes y\right)=\left(\phi x^{*} \otimes y\right) \text { for all } x^{*} \in X^{*}\right\},
$$

with equality for all $T \in \mathscr{B}\left(Y, X^{* *}\right)$ as above. It is easily seen that each $Y_{\Phi \phi}$ is a closed, possibly trivial, subspace of $Y$, and that if $\psi \in \mathrm{B}\left(X, X^{* *}\right)$ is another Hahn-Banach extension operator and $Y_{\Phi \phi} \cap Y_{\Phi \psi} \neq\{0\}$, then $\psi=\phi$.

Thus far we have shown that for every $z \in Z$ there is a $\phi_{z} \in \mathrm{B}\left(X, X^{* *}\right)$ such that $z \in Y_{\Phi \phi_{z}}$. Let us note that Lemma 1.1 in [13] remains true if we use it on

$$
Z_{\Phi \phi}=Z \cap Y_{\Phi \phi} .
$$


If $\left\{\phi_{i}: i \in I\right\}$ is a subset of $\mathrm{B}\left(X, X^{* *}\right)$, with all $\phi_{i}$ different, and if $z_{i} \in Z_{\Phi \phi_{i}}$ are such that $\sum_{i \in I} z_{i}=0$ and $\sum_{i \in I}\left\|z_{i}\right\|<\infty$, then $z_{i}=0$ for all $i \in I$ by Lemma 1.1 in [13].

Assume for contradiction that $\phi_{1}, \phi_{2} \in \mathrm{B}\left(X, X^{* *}\right)$ are different with $Z_{\Phi \phi_{1}}$ and $Z_{\Phi \phi_{2}}$ both non-trivial. Now pick $z_{i} \in Z_{\Phi \phi_{i}}, i=1,2$, with $z_{i} \neq 0$, $i=1,2$. Let $z_{3}=-\left(z_{1}+z_{2}\right)$. From the first part of the proof we see that there exists a $\phi_{3} \in \mathrm{H}\left(X, X^{* *}\right)$ such that $z_{3} \in Z_{\Phi \phi_{3}}$. If $\phi_{3} \neq \phi_{i}, i=1,2$, then $z_{1}=z_{2}=z_{3}=0$, a contradiction. If $\phi_{3}=\phi_{i}$, say $i=1$, then $z_{2}=$ $-\left(z_{3}+z_{1}\right) \in Z_{\Phi \phi_{1}} \cap Z_{\Phi \phi_{2}}=\{0\}$, so that $z_{2}=0$, again a contradiction.

This shows that all $\phi_{z}$, with $z \in Z$ and $Z_{\Phi \phi_{z}}$ non-trivial, are equal.

The next theorem is a generalization of Theorem 3.3 in [14].

THEOREM 2.4. Let $X$ and $Y$ be Banach spaces, and let $\mathscr{A}$ and $\mathscr{B}$ be closed operator ideals satisfying $\mathscr{A} \subseteq \mathscr{B}$. If $\mathscr{A}(\hat{Y}, X)$ is an ideal in $\mathscr{B}\left(\hat{Y}, X^{* *}\right)$ for all equivalent renormed versions $\hat{Y}$ of $Y$, then there exist a $\phi \in H\left(X, X^{* *}\right)$ and $a \Phi \in H\left(\mathscr{A}(Y, X), \mathscr{B}\left(Y, X^{* *}\right)\right)$ such that

$$
\Phi\left(x^{*} \otimes y\right)(T)=\left(\phi x^{*} \otimes y\right)(T)
$$

for all $x^{*} \in X^{*}, y \in Y$ and $T \in \mathscr{B}\left(Y, X^{* *}\right)$.

Proof. Let $Z$ be a separable subspace of $Y$. Proceeding as in the proof of Theorem 3.1 in [14], using Lemma 2.3, we find a $\Psi \in \operatorname{BB}\left(\mathscr{A}(Y, X), \mathscr{B}\left(Y, X^{* *}\right)\right)$ and a $\psi \in \mathrm{B}\left(X, X^{* *}\right)$ such that

$$
\Psi\left(x^{*} \otimes z\right)(T)=\left(\psi x^{*} \otimes z\right)(T)
$$

for all $x^{*} \in X^{*}, z \in Z$ and $T \in \mathscr{B}\left(Y, X^{* *}\right)$. Note that the proof of Theorem 3.1 in [14] refers in large parts to the proof of Theorem 2.3 from [13].

Arguing as in Lemma 3.2 in [14], we find that the set

$$
\begin{aligned}
K_{Z}=\left\{(\Psi, \psi) \in \mathrm{B}\left(\mathscr{A}(Y, X), \mathscr{B}\left(Y, X^{* *}\right)\right) \times \mathrm{B}\left(X, X^{* *}\right):\right. \\
\Psi\left(x^{*} \otimes z\right)(T)=\left(\psi x^{*} \otimes z\right)(T) \\
\text { for all } \left.x^{*} \in X^{*}, z \in Z, \text { and } T \in \mathscr{B}\left(Y, X^{* *}\right)\right\}
\end{aligned}
$$

is a subset of $\left(\left(\mathscr{B}\left(Y, X^{* *}\right) \hat{\otimes}_{\pi} \mathscr{A}(Y, X)^{*}\right)^{*} \times\left(X^{* *} \hat{\otimes}_{\pi} X^{*}\right)^{*}\right)$ which is compact in the product weak*-topology. From the proof of Theorem 3.3 in [14] it is now clear that there exist a $\phi \in \mathrm{B}\left(X, X^{* *}\right)$ and a $\Phi \in \mathrm{B}\left(\mathscr{A}(Y, X), \mathscr{B}\left(Y, X^{* *}\right)\right)$ such that

$$
\Phi\left(x^{*} \otimes y\right)(T)=\left(\phi x^{*} \otimes y\right)(T)
$$

for all $x^{*} \in X^{*}, y \in Y$, and $T \in \mathscr{B}\left(Y, X^{* *}\right)$. 
We will now show, given a Banach space $X$, that if $\mathscr{A}(Y, X)$ is an ideal in $\mathscr{B}\left(Y, X^{* *}\right)$ for all Banach spaces $Y$, then there is a $\phi \in \mathrm{B}\left(X, X^{* *}\right)$ which works for all $Y$. We shall soon make this precise (see Theorem 2.5), but first we introduce some notation. For each Banach space $Y$, let

$$
\begin{aligned}
& F_{Y}=\left\{\phi \in \mathrm{B}\left(X, X^{* *}\right): \text { there exists a } \Phi \in \mathrm{HB}\left(\mathscr{A}(Y, X), \mathscr{B}\left(Y, X^{* *}\right)\right)\right. \\
& \text { with } \Phi\left(x^{*} \otimes y\right)(T)=\left(\phi x^{*} \otimes y\right)(T) \\
& \text { for all } \left.x^{*} \in X^{*}, y \in Y, \text { and } T \in \mathscr{B}\left(Y, X^{* *}\right)\right\} .
\end{aligned}
$$

$F_{Y}$ is subset of $\mathscr{L}\left(X^{*}, X^{* * *}\right)=\left(X^{* *} \hat{\otimes}_{\pi} X^{*}\right)^{*}$. Theorem 2.4 shows that each $F_{Y}$ is nonempty whenever $\mathscr{A}(Y, X)$ is an ideal in $\mathscr{B}\left(Y, X^{* *}\right)$ for all Banach spaces $Y$. Theorem 2.3 in [13] shows that $F_{Y}$ is weak*-compact. The idea is now to show that the set $\left\{F_{Y}: Y\right.$ Banach space $\}$ has the finite intersection property.

The proof of our next theorem follows exactly along the lines of the proof of Theorem 2.4 in [13], which is similar, so we will not include it here.

Theorem 2.5. Let $X$ be a Banach space, and let $\mathscr{A}$ and $\mathscr{B}$ be closed operator ideals satisfying $\mathscr{A} \subseteq \mathscr{B}$. If $\mathscr{A}(Z, X)$ is an ideal in $\mathscr{B}\left(Z, X^{* *}\right)$ for all Banach spaces $Z$, then there exists a $\phi \in H\left(X, X^{* *}\right)$ such that, for every Banach space $Y$, there exists a $\Phi \in \boldsymbol{B}\left(\mathscr{A}(Y, X), \mathscr{B}\left(Y, X^{* *}\right)\right)$ with

$$
\Phi\left(x^{*} \otimes y\right)(T)=\left(\phi x^{*} \otimes y\right)(T)
$$

for all $x^{*} \in X^{*}, y \in Y$, and $T \in \mathscr{B}\left(Y, X^{* *}\right)$.

REMARK 2.6. It is clear, from the proofs and from [13], that in Lemma 2.3, Theorem 2.4, and Theorem 2.5 we may replace $X^{* *}$ with any Banach space containing $X$ as a closed subspace.

We conclude this section with the main result so far.

Theorem 2.7. Let $X$ be a Banach space. The following statements are equivalent.

(a) $X$ has the metric approximation property.

(b) There exists a $\phi \in \boldsymbol{H}\left(X, X^{* *}\right)$ such that, for every Banach space $Y$, there exists a $\Phi \in B\left(\mathscr{F}(Y, X), \mathscr{L}\left(Y, X^{* *}\right)\right)$ satisfying

$$
\Phi\left(x^{*} \otimes y\right)(T)=\left(\phi x^{*} \otimes y\right)(T)
$$

for all $x^{*} \in X^{*}, y \in Y$, and $T \in \mathscr{L}\left(Y, X^{* *}\right)$. 
(c) There exists $a \Phi \in H\left(\mathscr{F}(X, X)\right.$, $\left.\operatorname{span}\left(\mathscr{F}(X, X),\left\{I_{X}\right\}\right)\right)$ such that

$$
\Phi\left(x^{*} \otimes x\right)(T)=\left(x^{*} \otimes x\right)(T)
$$

for all $x \in X, x^{*} \in X^{*}$, and $T \in \operatorname{span}\left(\mathscr{F}(X, X),\left\{I_{X}\right\}\right)$.

Proof. Combining Lemma 2.1 and Theorem 2.5 we see that (a) $\Rightarrow$ (b). (b) $\Rightarrow$ (c) is trivial. (c) $\Rightarrow$ (a) is proved in [9] (cf. Theorem 5.4).

Remark 2.8. Theorem 2.7 remain valid for the metric compact approximation property if one replaces $\mathscr{F}(Y, X)$ with $\mathscr{K}(Y, X)$ and $\mathscr{F}(X, X)$ with $\mathscr{K}(X, X)$ (this is clear from the proof).

\section{Approximation properties for dual spaces}

In this section we prove that if we in Theorem 2.7 replace "there exists $\phi \in$ $\mathrm{HB}\left(X, X^{* *}\right)$ such that..." with "for all $\phi \in \mathrm{BB}\left(X, X^{* *}\right) \ldots$ " and require that the equality in Theorem 2.7 (b) holds for all $y^{* *} \in Y^{* *}$, not just all $y \in Y$, then we get a property which is equivalent to $X^{*}$ having the metric approximation property (see Theorem 3.10). We also obtain a similar "for all" characterization of the approximation property for $X^{*}$ (see Theorem 3.7).

In [12] (cf. Theorem 3.2) Lima and Oja obtained the following characterization of the approximation property for dual spaces.

Theorem 3.1. Let $X$ be a Banach space. The following statements are equivalent.

(a) $X^{*}$ has the approximation property.

(b) For all $\phi \in H \boldsymbol{B}\left(X, X^{* *}\right)$, all Banach spaces $Y$, and all $T \in \mathscr{W}\left(Y, X^{* *}\right)$ there exists a net $\left(T_{\alpha}\right) \subseteq \mathscr{F}(Y, X)$ with $\sup _{\alpha}\left\|T_{\alpha}\right\| \leq\|T\|$ such that $T_{\alpha}^{*} x^{*} \rightarrow T^{*}\left(\phi x^{*}\right)$ for all $x^{*} \in X^{*}$

(c) For all reflexive Banach spaces $Y$ and all $T \in \mathscr{K}\left(Y, X^{* *}\right)$ there exists a net $\left(T_{\alpha}\right) \subseteq \mathscr{F}(Y, X)$ with $\sup _{\alpha}\left\|T_{\alpha}\right\| \leq\|T\|$ such that $T_{\alpha}^{*} x^{*} \rightarrow T^{*} x^{*}$ for all $x^{*} \in X^{*}$.

Remark 3.2. Theorem 3.2 in [12] also states and proves that $X^{* *}$ in Theorem 3.1 may be replaced by any Banach space which contains $X$ as an ideal.

In [11] (cf. Theorem 4.6) it was proved that for a Banach space $X, \mathscr{F}(Y, X)$ is an ideal in $\mathscr{W}\left(Y, X^{* *}\right)$ with "nice" Hahn-Banach extension operators for all reflexive Banach spaces $Y$ whenever $X^{*}$ has the approximation property. We shall now remove this reflexivity condition using (b) in Theorem 3.1 to construct "nice" Hahn-Banach extension operators also for general $Y$. 
The next lemma shows that if (b) in Theorem 3.1 holds, then $\mathscr{F}(Y, X)$ is locally 1 -complemented in $\mathscr{W}\left(Y, X^{* *}\right)$ in a way that resembles the principle of local reflexivity.

Lemma 3.3. Let $X$ and $Y$ be Banach spaces and let $\phi \in H\left(X, X^{* *}\right)$. Let $G$ be a finite dimensional subspace of $\mathscr{W}\left(Y, X^{* *}\right)$, and let $H$ be a finite dimensional subspace of $\mathscr{F}(Y, X)^{*}$. If for any Banach space $Z$ and any $T \in$ $\mathscr{W}\left(Z, X^{* *}\right)$ there exists a net $\left(T_{\alpha}\right) \subseteq \mathscr{F}(Z, X)$ with $\sup _{\alpha}\left\|T_{\alpha}\right\| \leq\|T\|$ such that $T_{\alpha}^{*} x^{*} \rightarrow T^{*}\left(\phi x^{*}\right)$ for all $x^{*} \in X^{*}$, then for any $\varepsilon>0$ there exists a linear contraction $U: G \rightarrow \mathscr{F}(Y, X)$ such that

- $\|U(T)-T\|<\varepsilon\|T\|$ for all $T \in G \cap \mathscr{F}(Y, X)$, and

- $\left|\left(x^{*} \otimes y^{* *}\right)(U(T))-\left(\phi x^{*} \otimes y^{* *}\right)(T)\right| \leq \varepsilon\left\|x^{*} \otimes y^{* *}\right\|\|T\|$ for all $x^{*} \otimes y^{* *} \in$ $H$ and all $T \in G$.

Remark 3.4. As we will see from the proof we may replace $\mathscr{F}(Y, X)$ with $\mathscr{K}(Y, X)$ and $\mathscr{W}\left(Y, X^{* *}\right)$ with $\mathscr{L}\left(Y, X^{* *}\right)$. We may also replace $X^{* *}$ with any Banach space containing $X$ as an ideal.

Proof. Let $K_{G}=\overline{\operatorname{conv}}\left\{T\left(B_{Y}\right): T \in B_{G}\right\}$. Then $K_{G}$ is a closed absolutely convex subset of $B_{X^{* *}}$. Use the uniform isometric version of the Davis-FigielJohnson-Pełczyński factorization [9, Lemma 1.1] on $K_{G}$ to find a Banach space $Z$, a norm one operator $J: Z \rightarrow X^{* *}$, and a linear isometry $\Psi: G \rightarrow$ $\mathscr{W}(Y, Z)$ such that $T=J \circ \Psi(T)$ for all $T \in G$ (see Theorem 2.3 in [9]).

By assumption there exists a net $\left(J_{\alpha}\right) \subseteq \mathscr{F}(Z, X)$ with $\sup _{\alpha}\left\|J_{\alpha}\right\| \leq\|J\|$ such that

$$
J_{\alpha}^{*} x^{*} \longrightarrow J^{*}\left(\phi x^{*}\right)
$$

for all $x^{*} \in X^{*}$.

For $T \in G$, define $T_{\alpha}=J_{\alpha} \circ \Psi(T)$. Then we have

$$
\begin{aligned}
\lim _{\alpha}\left(x^{*} \otimes y^{* *}\right)\left(T_{\alpha}\right) & =\lim _{\alpha}\left\langle J_{\alpha}^{*} x^{*}, \Psi(T)^{* *} y^{* *}\right\rangle=\left\langle J^{*}\left(\phi x^{*}\right), \Psi(T)^{* *} y^{* *}\right\rangle \\
& =\left\langle\phi x^{*}, T^{* *} y^{* *}\right\rangle=\left(\phi x^{*} \otimes y^{* *}\right)(T)
\end{aligned}
$$

for all $y^{* *} \in Y^{* *}, x^{*} \in X^{*}$, and $T \in G$.

Let $F=G \cap \mathscr{F}(Y, X)$ and define

$$
K_{F}=\overline{\operatorname{conv}}\left\{T\left(B_{Y}\right): T \in B_{F}\right\} .
$$

Note that $K_{F}$ is norm compact in $X$ and in $Z$ (see Lemma 2.1 in [9]). If $x \in X \cap Z$, then we get

$$
\left\langle J_{\alpha} x, x^{*}\right\rangle=\left\langle x, J_{\alpha}^{*} x^{*}\right\rangle \rightarrow_{\alpha}\left\langle x, J^{*}\left(\phi x^{*}\right)\right\rangle=\left\langle J x, \phi x^{*}\right\rangle=\left\langle J x, x^{*}\right\rangle,
$$


which means that $\left.\left.J_{\alpha}\right|_{X \cap Z} \rightarrow J\right|_{X \cap Z}$ in the weak operator topology. By choosing a new net, still denoted $\left(J_{\alpha}\right)$, of convex combination of $\left(J_{\alpha}\right)$ we may assume that $\left.\left.J_{\alpha}\right|_{X \cap Z} \rightarrow J\right|_{X \cap Z}$ in the strong operator topology. Since $\left(J_{\alpha}\right)$ is bounded, $J_{\alpha} \rightarrow J$ uniformly on $K_{F}$.

Choosing $\alpha_{0}$ large enough and defining $U(T)=J_{\alpha_{0}} \circ \Psi(T)$ for $T \in G$, we get the desired operator.

Using the Lindenstrauss compactness principle with ideas from [4] (see also [5]) we will now construct a Hahn-Banach extension operator with nice properties from the "locally nice" extensions in Lemma 3.3.

Lemma 3.5. Let $X$ and $Y$ be Banach spaces, and let $\phi \in H\left(X, X^{* *}\right)$. If for every pair of finite dimensional subspaces $G \subseteq \mathscr{W}\left(Y, X^{* *}\right)$ and $H \subseteq$ $\mathscr{F}(Y, X)^{*}$ and every $\varepsilon>0$ there exists a linear contraction $U$ as in Lemma 3.3 then there exists a $\Phi \in B\left(\mathscr{F}(Y, X), \mathscr{W}\left(Y, X^{* *}\right)\right)$ such that

$$
\Phi\left(x^{*} \otimes y^{* *}\right)(T)=\left(\phi x^{*} \otimes y^{* *}\right)(T)
$$

for all $y^{* *} \in Y^{* *}, x^{*} \in X^{*}$, and $T \in \mathscr{W}\left(Y, X^{* *}\right)$.

Proof. Let $H \subseteq \mathscr{F}(Y, X)^{*}$ be a finite dimensional subspace. For each finite dimensional subspace $G \subseteq \mathscr{W}\left(Y, X^{* *}\right)$, let $\varepsilon=1 / \operatorname{dim} G$ and choose a contractive operator $U_{G}: G \rightarrow \mathscr{F}(Y, X)$ such that

- $\left\|U_{G}(T)-T\right\|<\varepsilon\|T\|$ for all $T \in G \cap \mathscr{F}(Y, X)$, and

- $\left|\left(x^{*} \otimes y^{* *}\right)\left(U_{G}(T)\right)-\left(\phi x^{*} \otimes y^{* *}\right)(T)\right| \leq \varepsilon\left\|x^{*} \otimes y^{* *}\right\|\|T\|$ for all $x^{*} \otimes y^{* *} \in H$ and all $T \in G$.

Note that $\left|\psi\left(U_{G}(T)\right)-\psi(T)\right|<\varepsilon\|\psi\|\|T\|$ for all $\psi \in H$ and all $T \in$ $G \cap \mathscr{F}(Y, X)$.

Extend $U_{G}: \mathscr{W}\left(Y, X^{* *}\right) \rightarrow \mathscr{F}(Y, X)$ by letting $U_{G}(T)=0$ if $T \notin G$. Define

$$
\widetilde{\Xi}_{H}=\prod_{T \in \mathscr{W}\left(Y, X^{* *}\right)} B_{\mathscr{F}(Y, X) / H_{\perp}}(0,\|T\|) .
$$

$\mathscr{F}(Y, X) / H_{\perp}$ is finite dimensional so $\Xi_{H}$ is a compact Hausdorff space when equipped with the product topology. Let $q_{H}: \mathscr{F}(Y, X) \rightarrow \mathscr{F}(Y, X) / H_{\perp}$ be the quotient mapping and define a net in $\widetilde{\Xi}_{H}$ by $\left(q_{H} U_{G}(T)\right)_{G}$ (ordered by inclusion). By compactness we may find a convergent subnet, still denoted $\left(q_{H} U_{G}(T)\right)_{G}$. Define

$$
Q_{H}: \mathscr{W}\left(Y, X^{* *}\right) \longrightarrow \mathscr{F}(Y, X) / H_{\perp}
$$

by $Q_{H}(T)=\lim _{G} q_{H} U_{G}(T) . Q_{H}$ is linear and has norm one, and using the assumptions on $U_{G}$ we get

$$
Q_{H}(T)=\lim _{G} q_{H} U_{G}(T)=q_{H}(T)
$$


for all $T \in \mathscr{F}(Y, X)$. This last equality can be written $q_{H}=Q_{H} \circ i$, where $i: \mathscr{F}(Y, X) \rightarrow \mathscr{W}\left(Y, X^{* *}\right)$ is the natural inclusion. From this we see that $i^{*} \circ Q_{H}^{*}=q_{H}^{*}$, so that $Q_{H}^{*}: H \rightarrow \mathscr{W}\left(Y, X^{* *}\right)^{*}$ is a Hahn-Banach extension operator. Furthermore, if $\psi=x^{*} \otimes y^{* *} \in H$ and $T \in \mathscr{W}\left(Y, X^{* *}\right)$, then

$$
\begin{aligned}
\left(Q_{H}^{*} \psi\right)(T) & =\psi\left(Q_{H}(T)\right)=\lim _{G} \psi\left(q_{H} U_{G}(T)\right) \\
& =\lim _{G}\left(q_{H}^{*} \psi\right)\left(U_{G}(T)\right)=\lim _{G}\left(x^{*} \otimes y^{* *}\right)\left(U_{G}(T)\right) \\
& =\left(\phi x^{*} \otimes y^{* *}\right)(T)
\end{aligned}
$$

since $\lim _{G}\left|\left(x^{*} \otimes y^{* *}\right)\left(U_{G} T\right)-\left(\phi x^{*} \otimes y^{* *}\right)(T)\right|=0$.

Extend $Q_{H}^{*}: \mathscr{F}(Y, X)^{*} \rightarrow \mathscr{W}\left(Y, X^{* *}\right)^{*}$ by letting $Q_{H}^{*}(\psi)=0$ if $\psi \notin H$. Let

$$
\widetilde{\subseteq}=\prod_{\psi \in \mathscr{F}(Y, X)^{*}} B \mathscr{W}\left(Y, X^{* *}\right)^{*}(0,\|\psi\|)
$$

$\widetilde{\varsigma}$ is compact and Hausdorff when equipped with the product weak* topology. $\left(Q_{H}^{*} \psi\right)_{H}$ is a net in $\subseteq$ (ordered by inclusion), so it has a subnet converging to a Hahn-Banach extension operator $\Phi: \mathscr{F}(Y, X)^{*} \rightarrow \mathscr{W}\left(Y, X^{* *}\right)^{*}$ such that

$$
\Phi\left(x^{*} \otimes y^{* *}\right)(T)=\lim _{H}\left(Q_{H}^{*} \psi\right)(T)=\left(x^{*} \otimes y^{* *}\right)(T) .
$$

REMARK 3.6. The above lemma still holds if we replace $\mathscr{F}$ and $\mathscr{W}$ with any pair of operator ideals $\mathscr{A}$ and $\mathscr{B}$, such that $\mathscr{A} \subseteq \mathscr{B}$. We may also replace $X^{* *}$ with any Banach space containing $X$ as an ideal.

Combining Theorem 3.1 with Lemmas 3.3 and 3.5 gives the following characterization of the approximation property for dual spaces in terms of "nice" Hahn-Banach extension operators.

THEOREM 3.7. Let $X$ be a Banach space. The following statements are equivalent.

(a) $X^{*}$ has the approximation property.

(b) For all $\phi \in H\left(X, X^{* *}\right)$ and all Banach spaces $Y$ there exists a $\Phi \in$ $H\left(\mathscr{F}(Y, X), \mathscr{W}\left(Y, X^{* *}\right)\right)$ such that

$$
\Phi\left(x^{*} \otimes y^{* *}\right)(T)=\left(\phi x^{*} \otimes y^{* *}\right)(T)
$$

for all $x^{*} \in X^{*}, y^{* *} \in Y^{* *}$, and $T \in \mathscr{W}\left(Y, X^{* *}\right)$.

(c) For all reflexive Banach spaces $Y$ there is a $\Phi \in B\left(\mathscr{F}(Y, X), \mathscr{K}\left(Y, X^{* *}\right)\right)$ such that

$$
\Phi\left(x^{*} \otimes y\right)(T)=\left(x^{*} \otimes y\right)(T)
$$


for all $x^{*} \in X^{*}, y \in Y$, and $T \in \mathscr{K}\left(Y, X^{* *}\right)$.

Proof. (a) $\Rightarrow$ (b). Assume that $X^{*}$ has the approximation property. From Theorem 3.1 (b) we see that the assumption of Lemma 3.3 is fulfilled. The assumption in Lemma 3.5 is the conclusion of Lemma 3.3 and the conclusion of Lemma 3.5 is (b).

(b) $\Rightarrow$ (c) is trivial.

(c) $\Rightarrow$ (a). Proceeding as in Lemma 1.4 in [9], we define a projection $P$ by $P=\Phi \circ i^{*}$, where $i: \mathscr{F}(Y, X) \rightarrow \mathscr{K}\left(Y, X^{* *}\right)$ is the natural embedding. For every $T \in \mathscr{K}\left(Y, X^{* *}\right)$ we have $P^{*}(T) \in \mathscr{F}(Y, X)^{\perp \perp}$, so there is a net $\left(T_{\alpha}\right) \subseteq \mathscr{F}(Y, X)$ converging weak* to $P^{*}(T)$ from below. It is easily seen that this implies condition (c) in Theorem 3.1.

Remark 3.8. From Remarks 3.2, 3.4, and 3.6 we see that in the above theorem $X^{* *}$ may be replaced by any Banach space containing $X$ as an ideal.

As the following example shows, the "for all $\phi$ "-part in Theorem 3.7 is necessary. Although we work with a concrete space in the example the essential ingredient is the existence of a Banach space $X$ with the metric approximation property such that $X^{*}$ does not have the approximation property.

Example 3.9. Let $X=\ell_{2} \hat{\otimes}_{\pi} \ell_{2}$. The Banach space $X$ satisfies the following.

(i) There exists a $\phi \in \mathrm{B}\left(X, X^{* *}\right)$ with the property that for every Banach space $Y$ there exists a $\Phi \in \mathrm{B}\left(\mathscr{F}(Y, X), \mathscr{W}\left(Y, X^{* *}\right)\right)$ such that

$$
\Phi\left(x^{*} \otimes y\right)(T)=\left(\phi x^{*} \otimes y\right)(T)
$$

for all $x^{*} \in X^{*}, y \in Y$, and $T \in \mathscr{W}\left(Y, X^{* *}\right)$.

(ii) (i) is not satisfied for every $\phi \in \mathrm{B}\left(X, X^{* *}\right)$. In particular, $\phi$ cannot be the natural embedding of $X^{*}$ into $X^{* * *}$.

Proof. It is well-known that $X$ has the metric approximation property $\left(X=\left(\ell_{2} \hat{\otimes}_{\varepsilon} \ell_{2}\right)^{*}\right.$ is a dual space with a basis).

By Theorem 2.7, there exists a $\phi \in \mathrm{BB}\left(X, X^{* *}\right)$ satisfying (i). Theorem 3.7 above says that $X^{*}$ has the approximation property if and only if such a $\Phi$ exists for all $\phi \in \mathrm{B}\left(X, X^{* *}\right)$ or just for the natural embedding. However, in [18] Szankowski showed that $X^{*}=\mathscr{L}\left(\ell_{2}, \ell_{2}\right)$ does not have the approximation property, and thus we have (ii).

We conclude this section with a metric version of Theorems 3.1 and 3.7 complementing Theorem 2.7. The next theorem should also be compared with the version of Theorem 5.2 in [9] cited in the Introduction. 
THEOREM 3.10. Let $X$ be a Banach space. The following statements are equivalent.

(a) $X^{*}$ has the metric approximation property

(b) For all Banach spaces $Y$ and all $\phi \in H\left(X, X^{* *}\right)$ there exists $a \Phi \in$ $H\left(\mathscr{F}(Y, X), \mathscr{L}\left(Y, X^{* *}\right)\right)$ such that

$$
\Phi\left(x^{*} \otimes y^{* *}\right)(T)=\left(\phi x^{*} \otimes y^{* *}\right)(T)
$$

for all $x^{*} \in X^{*}, y^{* *} \in Y^{* *}$, and $T \in \mathscr{L}\left(Y, X^{* *}\right)$.

(c) For all Banach spaces $Y$ there exists $a \Phi \in H\left(\mathscr{F}(Y, X), \mathscr{L}\left(Y, X^{* *}\right)\right)$ such that

$$
\Phi\left(x^{*} \otimes y^{* *}\right)(T)=\left(x^{*} \otimes y^{* *}\right)(T)
$$

for all $x^{*} \in X^{*}, y^{* *} \in Y^{* *}$, and $T \in \mathscr{L}\left(Y, X^{* *}\right)$.

(d) For all Banach spaces $Y$, all $\phi \in H\left(X, X^{* *}\right)$, and all $T \in \mathscr{L}\left(Y, X^{* *}\right)$ there exists a net $\left(T_{\alpha}\right) \subseteq \mathscr{F}(Y, X)$ with $\sup _{\alpha}\left\|T_{\alpha}\right\| \leq\|T\|$ such that $T_{\alpha}^{*} x^{*} \rightarrow T^{*}\left(\phi x^{*}\right)$ for all $x^{*} \in X^{*}$.

(e) For all Banach spaces $Y$ and all $T \in \mathscr{L}\left(Y, X^{* *}\right)$ there exists a net $\left(T_{\alpha}\right) \subseteq \mathscr{F}(Y, X)$ with $\sup _{\alpha}\left\|T_{\alpha}\right\| \leq\|T\|$ such that $T_{\alpha}^{*} x^{*} \rightarrow T^{*} x^{*}$ for all $x^{*} \in X^{*}$.

Proof. (a) $\Rightarrow$ (d). Let $\left(S_{\alpha}\right) \subseteq \mathscr{F}(X, X)$ with $\sup _{\alpha}\left\|S_{\alpha}\right\|=1$ such that $S_{\alpha}^{*} x^{*} \rightarrow x^{*}$ for all $x^{*} \in X^{*}$. Let $\phi \in \mathrm{BB}\left(X, X^{* *}\right)$ and $T \in \mathscr{L}\left(Y, X^{* *}\right)$, then $\phi^{*} \circ T \in \mathscr{L}\left(Y, X^{* *}\right)$. Define $T_{\alpha}=S_{\alpha} \circ \phi^{*} \circ T$. For $x^{*} \in X^{*}$ we get

$$
T_{\alpha}^{*} x^{*}=\left(\phi^{*} T\right)^{*} S_{\alpha}^{*} x^{*} \longrightarrow\left(\phi^{*} T\right)^{*} x^{*}=T^{*}\left(\phi x^{*}\right) .
$$

(b) $\Rightarrow$ (c) and (d) $\Rightarrow$ (e) are trivial.

(e) $\Rightarrow$ (a). Let $Y=X$ and $T=\left.\left(I_{X^{*}}^{*}\right)\right|_{X}$. Then there exists a net $\left(T_{\alpha}\right) \subseteq$ $\mathscr{F}(X, X)$ with $\sup _{\alpha}\left\|T_{\alpha}\right\| \leq\|T\|=1$ such that

$$
T_{\alpha}^{*} x^{*} \longrightarrow T^{*} x^{*}=x^{*} .
$$

(c) $\Rightarrow$ (e). Argue as in the proof of (c) $\Rightarrow$ (a) in Theorem 3.7.

Finally, to show (d) $\Rightarrow$ (b) we use Lemmas 3.3 and 3.5 (see also Remarks 3.4 and 3.6).

Remark 3.11. Replacing $\mathscr{F}(Y, X)$ with $\mathscr{K}(Y, X)$ in the above theorem we get a characterization of the metric compact approximation property with conjugate operators for dual spaces.

It should be noted that thanks to the principle of local reflexivity the metric approximation property and the metric approximation property with conjugate operators are equivalent. 
REMARK 3.12. A Banach space $X$ is said to have the unique extension property if $\mathrm{B}\left(X, X^{* *}\right)=\{i\}$, where $i$ is the natural embedding $i: X^{*} \rightarrow X^{* * *}$. In [3] Godefroy and Saphar proved that if $X$ has the unique extension property and the metric approximation property, then $X^{*}$ has the metric approximation property. This result should be compared with Theorems 2.7 and 3.10, but it is, however, not implied by these two theorems.

\section{Renorming and extension operators}

It was shown in [9], relying on [10], that a Banach space $X$ has the approximation property if and only if $\mathscr{F}(Y, X)$ is an ideal in $\mathscr{W}(Y, X)$ for every Banach space $Y$. In Theorem 4.5 we will prove that $X^{*}$ has the approximation property if and only if $\mathscr{F}(Y, \hat{X})$ is an ideal in $\mathscr{W}\left(Y, \hat{X}^{* *}\right)$ for all Banach spaces $Y$ and all equivalent renormings $\hat{X}$ of $X$. It follows that a result similar to Lemma 2.1 cannot be true for the approximation property. That is, $X$ has the approximation property does not imply that $\mathscr{F}(Y, X)$ is an ideal in $\mathscr{W}\left(Y, X^{* *}\right)$ for every Banach space $Y$. If it did, then the fact that $X$ does not loose the approximation property under an equivalent norm together with Theorem 4.5 would imply that $X^{*}$ had the approximation property. This is not the case (see e.g. Theorem 1.e.7(b) in [15]).

To show the above characterization of the approximation property for dual spaces we will be using ideas from [14]. As in Theorem 3.7 above we want to know what the Hahn-Banach extensions of simple tensors look like. This next lemma is a crucial step toward this goal, and it is actually a special case of Lemma 3.1 in [11].

Lemma 4.1. Let $X$ and $Y$ be Banach spaces, and let $\mathscr{A}$ and $\mathscr{B}$, where $\mathscr{A} \subseteq \mathscr{B}$, be a pair of closed operator ideals. If the norm on $X^{*}$ is locally uniformly rotund at $x^{*} \in X^{*}$ and $y^{* *} \in Y^{* *}$, then $x^{*} \otimes y^{* *} \in \mathscr{A}(Y, X)^{*}$ has a unique extension to $\mathscr{B}\left(Y, X^{* *}\right)$, i.e.

$$
H\left(x^{*} \otimes y^{* *}\right)=\left\{x^{*} \otimes y^{* *}\right\} .
$$

Proof. The local uniform rotundity of the norm of $X^{*}$ at $x^{*} \in S_{X^{*}}$ implies that $x^{*}$ is a weak ${ }^{*}$-denting point in $B_{X^{*}}$, so we may use Lemma 3.1 in [11].

THEOREM 4.2. Let $X$ and $Y$ be Banach spaces and assume that $\mathscr{A}(Y, \hat{X})$ is an ideal in $\mathscr{B}\left(Y, \hat{X}^{* *}\right)$ for all equivalent renormings $\hat{X}$ of $X$. If $F$ is a finite dimensional subspace of $X^{*}$, then there exists a $\Psi \in H\left(\mathscr{A}(Y, X), \mathscr{B}\left(Y, X^{* *}\right)\right)$ such that

$$
\Psi\left(x^{*} \otimes y^{* *}\right)(T)=\left(x^{*} \otimes y^{* *}\right)(T)
$$

for all $x^{*} \in F, y^{* *} \in Y^{* *}$ and $T \in \mathscr{B}\left(Y, X^{* *}\right)$. 
Proof. Let $F$ be a finite dimensional subspace of $X^{*}$. For all $\varepsilon \in(0,1]$ there exists an equivalent norm $\|\cdot\|_{\varepsilon}$ on $X$ such that the dual norm on $X^{*}$ is locally uniformly rotund at every point $x^{*}$ in $F$ and such that

$$
B_{X}(0,1) \subseteq B_{\left(X,\|\cdot\|_{\varepsilon}\right)}(0,1) \subseteq B_{X}(0,1+\varepsilon),
$$

by Lemma 2.4 in [14]. Let $X_{\varepsilon}=\left(X,\|\cdot\|_{\varepsilon}\right)$.

By assumption $\mathscr{A}\left(Y, X_{\varepsilon}\right)$ is an ideal in $\mathscr{B}\left(Y, X_{\varepsilon}^{* *}\right)$ so there exists a HahnBanach extension operator $\Phi_{\varepsilon}: \mathscr{A}\left(Y, X_{\varepsilon}\right)^{*} \rightarrow \mathscr{B}\left(Y, X_{\varepsilon}^{* *}\right)^{*}$. From Lemma 4.1 we get

$$
\Phi_{\varepsilon}\left(x^{*} \otimes y^{* *}\right)(T)=\left(x^{*} \otimes y^{* *}\right)(T)
$$

for all $x^{*} \in F_{\varepsilon}, y^{* *} \in Y^{* *}$, and $T \in \mathscr{B}\left(Y, X_{\varepsilon}^{* *}\right)$.

Let $I_{\varepsilon}: X_{\varepsilon} \rightarrow X$ denote the identity mapping. Then $\left\|I_{\varepsilon}^{-1}\right\|=1$ and $\left\|I_{\varepsilon}\right\| \rightarrow 1$ as $\varepsilon \rightarrow 0$. Define $\Psi_{\varepsilon} \in \mathscr{L}\left(\mathscr{A}(Y, X)^{*}, \mathscr{B}\left(Y, X^{* *}\right)^{*}\right)$ by

$$
\left(\Psi_{\varepsilon} g\right)(T)=\left(\Phi_{\varepsilon} g_{\varepsilon}\right)\left(\left(I_{\varepsilon}^{* *}\right)^{-1} \circ T\right)
$$

for $g \in \mathscr{A}(Y, X)^{*}$ and $T \in \mathscr{B}\left(Y, X^{* *}\right)$, and where $g_{\varepsilon} \in \mathscr{A}\left(Y, X_{\varepsilon}\right)^{*}$ is defined by

$$
g_{\varepsilon}(S)=g\left(I_{\varepsilon} \circ S\right)
$$

for $S \in \mathscr{A}\left(Y, X_{\varepsilon}\right)$. We may now proceed as in [13, Theorem 2.3] to show that $\left(\Psi_{\varepsilon}\right)_{\varepsilon \in(0,1]}$ has a subnet which converges weak ${ }^{*}$ to the desired $\Psi$.

Lemma 4.3. Let $X$ and $Y$ be Banach spaces and let $F$ be a finite dimensional subspace of $X^{*}$. The subset

$$
\begin{array}{r}
K_{F}=\left\{\Psi \in H\left(\mathscr{A}(Y, X), \mathscr{B}\left(Y, X^{* *}\right)\right): \Psi\left(x^{*} \otimes y^{* *}\right)(T)=\left(x^{*} \otimes y^{* *}\right)(T),\right. \\
\text { for all } \left.x^{*} \in F, y^{* *} \in Y^{* *}, T \in \mathscr{B}\left(Y, X^{* *}\right)\right\}
\end{array}
$$

of $\left(\mathscr{B}\left(Y, X^{* *}\right) \hat{\otimes}_{\pi} \mathscr{A}(Y, X)^{*}\right)^{*}$ is weak ${ }^{*}$-compact.

Proof. The proof is similar to the proof of Lemma 4.2 in [14].

Theorem 4.4. Let $X$ and $Y$ be Banach spaces. If $\mathscr{A}(Y, \hat{X})$ is an ideal in $\mathscr{B}\left(Y, \hat{X}^{* *}\right)$ for all equivalent renormings $\hat{X}$ of $X$ then there exists a $\Psi \in$ $H\left(\mathscr{A}(Y, X), \mathscr{B}\left(Y, X^{* *}\right)\right)$ such that

$$
\Psi\left(x^{*} \otimes y^{* *}\right)(T)=\left(x^{*} \otimes y^{* *}\right)(T)
$$

for all $x^{*} \in X^{*}, y^{* *} \in Y^{* *}$, and $T \in \mathscr{B}\left(Y, X^{* *}\right)$.

Proof. The proof is similar to the proof of Theorem 4.3 in [14].

Theorem 4.5. Let $X$ be a Banach space. The following statements are equivalent. 
(a) $X^{*}$ has the approximation property.

(b) For all Banach spaces $Y$ and all equivalent renormings $\hat{X}$ of $X, \mathscr{F}(Y, \hat{X})$ is an ideal in $\mathscr{W}\left(Y, \hat{X}^{* *}\right)$.

(c) For all reflexive Banach spaces $Y$ and all equivalent renormings $\hat{X}$ of $X, \mathscr{F}(Y, \hat{X})$ is an ideal in $\mathscr{K}\left(Y, \hat{X}^{* *}\right)$.

Proof. (a) $\Rightarrow$ (b). If $X^{*}$ has the approximation property, then $\hat{X}^{*}$ has the approximation property for every equivalent renorming $\hat{X}$ of $X$. Using Corollary 4.7 in [11], we get that $\mathscr{F}(Y, \hat{X})$ is an ideal in $\mathscr{W}\left(Y, \hat{X}^{* *}\right)$ for all Banach spaces $Y$.

(b) $\Rightarrow$ (c) is trivial. Combining Theorems 3.7 and 4.4 we see that (c) $\Rightarrow$ (a).

Remark 4.6. We have already noted that a result similar to Lemma 2.1 cannot be true for the approximation property. There are Banach spaces $X$ with the metric approximation property such that their duals do not have the approximation property, Example 3.9 provides one example of such a space. Thus it follows that the property " $\mathscr{F}(Y, X)$ is an ideal in $\mathscr{W}\left(Y, X^{* *}\right)$ for all Banach spaces $Y$ " is strictly between " $X$ has the approximation property" and " $X^{*}$ has the approximation property".

It should also be noted that Example 4.1 in [12] shows that $\mathscr{F}(Y, X)$ being an ideal in $\mathscr{K}\left(Y, X^{* *}\right)$ for all Banach spaces $Y$ does not imply the approximation property for $X^{*}$. However, if $X$ has the unique extension property then the two are in fact equivalent (cf. [12, Corollary 4.2]).

AcKNOWLEDGEMEnTs. The author wishes to thank E. Oja for several helpful suggestions.

\section{REFERENCES}

1. Fakhoury, H., Sélections linéaires associées au théorème de Hahn-Banach, J. Funct. Anal. 11 (1972), 436-452.

2. Godefroy, G., Kalton, N. J., and Saphar, P. D., Unconditional ideals in Banach spaces, Studia Math. 104 (1993), 13-59.

3. Godefroy, G., and Saphar, P., Duality in spaces of operators and smooth norms on Banach spaces, Illinois J. Math. 32 (1988), 672-695.

4. González, M., and Martínez-Abejón, A., Local dual spaces of a Banach space, Studia Math. 147 (2001), no. 2, 155-168. MR 1855821

5. Heinrich, S., Ultraproducts in Banach space theory, J. Reine Angew. Math. 313 (1980), 72-104.

6. Johnson, J., Remarks on Banach spaces of compact operators, J. Funct. Anal. 32 (1979), 304-311.

7. Kalton, N. J., Locally complemented subspaces and $\mathscr{L}_{p}$-spaces for $0<p<1$, Math. Nachr. 115 (1984), 71-97.MR 86h:46006

8. Lima, Å, The metric approximation property, norm-one projections and intersection properties of balls, Israel J. Math. 84 (1993), 451-475. 
9. Lima, $\AA$, Nygaard, O., and Oja, E., Isometric factorization of weakly compact operators and the approximation property, Israel J. Math. 119 (2000), 325-348.

10. Lima, $\AA$, and Oja, E., Ideals of finite rank operators, intersection properties of balls, and the approximation property, Studia Math. 133 (1999), no. 2, 175-186. MR 2000c:46026

11. Lima, Å, and Oja, E., Ideals of compact operators, J. Austral. Math. Soc. (to appear).

12. Lima, Å, and Oja, E., Ideals of operators, approximability in the strong operator topology, and the approximation property, Submitted, 2001.

13. Lima, $\AA$, and Oja, E., Hahn-Banach extension operators and spaces of operators, Proc. Amer. Math. Soc. 130 (2002), 3631-3640.

14. Lima, V., and Lima, Å, Ideals of operators and the metric approximation property, J. Funct. Anal. (to appear).

15. Lindenstrauss, J., and Tzafriri, L., Classical Banach Spaces I, Springer, Berlin-HeidelbergNew York, 1977.

16. Megginson, R. E., An Introduction to Banach Space Theory, Springer-Verlag, New York, 1998. MR 99k:46002

17. Pietsch, A., Operator Ideals, North Holland, Amsterdam-New York-Oxford, 1980.

18. Szankowski, A., $B(H)$ does not have the approximation property, Acta Math. 147 (1981), no. 1-2, 89-108. MR 83a:46033

DEPARTMENT OF MATHEMATICS

AGDER COLLEGE

SERVICEBOKS 422

4604 KRISTIANSAND

NORWAY

E-mail: Vegard.Lima@hia.no 